\title{
On the form of the H II region luminosity function
}

Accepted 10-Dec-97 to the Astronomical Journal

\author{
M. S. Oey and C. J. Clarke \\ Institute of Astronomy, Madingley Road, Cambridge, CB3 0HA, U.K. \\ oey@ast.cam.ac.uk; cclarke@ast.cam.ac.uk
}

\begin{abstract}
Observed variations in the $\mathrm{H}$ II region luminosity function ( $\mathrm{H}$ II LF) seen in spiral arm vs. interarm regions, and different galactic Hubble type, can be explained simply by evolutionary effects and maximum number of ionizing stars per cluster. We present Monte Carlo simulations of the H II LF, drawing the number of ionizing stars $N_{*}$ from a power-law distribution of constant slope, and the stellar masses from a Salpeter IMF with an upper-mass limit of $100 \mathrm{M}_{\odot}$. We investigate the evolution of the HII LF, as determined by stellar main-sequence lifetimes and ionizing luminosities, for a single burst case and continuous creation of the nebular population. Shallower H II LF slopes measured for the arms of spiral galaxies can be explained as a composite slope, expected for a zero-age burst population, whereas the interarm regions tend to be dominated by evolved rich clusters described by a single, steeper slope. Steeper slopes in earlier-type galaxies can be explained simply by a lower maximum $N_{*}$ cutoff found for the parent OB associations. The form of the H II LF can reveal features of the most recent $(\lesssim 10$ Myr) star formation history in nearby galaxies.
\end{abstract}

Subject headings: H II regions — galaxies: fundamental parameters — galaxies: ISM galaxies: star clusters - ISM: structure — stars: formation 


\section{Introduction}

$\mathrm{H}$ II regions have long served as a primary indicator of high-mass star formation, both in the Milky Way and in external galaxies. The luminosity function of $\mathrm{H}$ II regions (H II LF) is therefore a vital probe of the present global star formation properties in individual galaxies. In recent years, the number and accuracy of compiled H II LFs for nearby galaxies has increased substantially, extending the use of this tool to greater distances.

The differential H II LF is usually parameterized as a power law:

$$
N(L) d L=A L^{-a} d L
$$

where $N(L) d L$ is the number of nebulae with luminosities in the range $L$ to $L+d L$. Some interesting patterns have emerged regarding the form of the H II LF:

1. A large number of galaxies show a break in slope of the HII LF with the fainter H II regions showing a shallower slope compared to the high-luminosity objects (e.g., Kennicutt, Edgar, \& Hodge 1989, hereafter KEH; Rand 1992; Walterbos \& Braun 1992; Rozas, Beckman, \& Knapen 1996a;)

2. The HII LF for arm and interarm regions of spiral galaxies sometimes show steeper slopes in the interarm regions (e.g., KEH; Banfi et al. 1993; Rand 1992). However, there are also many instances where no difference is found (e.g., Rozas et al. 1996a; Knapen et al. 1993; Knapen 1997)

3. The slope of the H II LF is correlated with galactic Hubble type, such that early-type galaxies show steeper slopes than late-types. The powerlaw index $a \sim 2.0$ for $\mathrm{Sb}-\mathrm{Sc}$ galaxies, compared to $a \sim 1.7$ for Sc - Im galaxies (KEH; Banfi et al. 1993). It is even steeper in Sa galaxies, with $a \sim 2.6$ (Caldwell et al. 1991).

These differences in the HII LF have usually been interpreted as resulting from corresponding differences in star formation properties. For example, the break in slope (Point 1, above) has been suggested to be caused by a physical transition between normal $\mathrm{H}$ II regions and the class of supergiant H II regions such as 30 Doradus in the Large Magellanic Cloud (KEH). Likewise, the difference in slope for arm vs. interarm regions, and among various Hubble types (Points 2 and 3), have been attributed to differences in gasdynamics and molecular cloud mass spectrum (e.g., Thronson, Rubin, and Ksir 1991; Rand 1992; $\mathrm{KEH})$. However, we feel that it may be premature to draw a direct connection between the form of the H II LF and details of star forming environment such as these. There are other effects that can influence the form of the HII LF, that have not been adequately explored.

One important effect, in particular, is the influence of simple evolution in the ionizing clusters, and hence, luminosity of the host H II regions. There are several empirical factors that suggest that nebular luminosity evolution is an important effect in the form of the H II LF. The steeper slopes seen in interarm regions of spiral galaxies (Point 2, above) have been identified by von Hippel \& Bothun (1990) as evidence of an aging effect in those populations. Assuming star formation takes place primarily in the spiral density waves delineated by the arm regions, then the interarm regions should exhibit a typically older population of nebulae in the wake of this star formation activity. In addition, von Hippel \& Bothun cite the behavior of $\mathrm{H} \alpha$ equivalent widths as further compelling evidence of the importance of luminosity evolution. As a cluster ages, its associated $\mathrm{H} \alpha$ emission decreases, while that of the underlying red stellar continuum increases, thereby reducing the $\mathrm{H} \alpha$ equivalent width as a function of time. They demonstrate that for the H II regions in NGC 628, there is indeed a clear decrease in $\mathrm{H} \alpha$ equivalent width with decreasing $\mathrm{H} \alpha$ luminosity, indicating higher numbers of evolved nebulae among the fainter populations. Finally, Knapen et al. (1993) and Rozas, Knapen, \& Beckman (1996b) show that the slope of the nebular luminosity vs. volume relation is slightly flatter in the interarm regions of five galaxies as compared to the arm regions. As emphasized by the authors, this may be caused by observational selection effects, but if real, we suggest that it is consistent with a higher tendency for shell-like geometries in the interarm nebulae, as would be expected for an older population.

All of these arguments suggest that evolved nebulae will be an important population in the H II LF. Von Hippel \& Bothun (1990) carried out the first detailed investigation of the evolutionary effect on the H II LF. They constructed two models to reproduce the observed slope for NGC 628: one with zero evolution, in which the power-law slope of the luminosity 
function is due entirely to the cluster mass spectrum; and one in which the distribution in nebular luminosity is due entirely to a different age and number distribution of objects with the same initial cluster mass. The H II LF resulting from the evolutionary model is sensitive to the stellar initial mass function (IMF), since the relative numbers of stars at a given ionizing luminosity become important when these stars become the dominant contributor to the nebular luminosity. Recognizing this, von Hippel \& Bothun oriented their study toward exploring the use of the H II LF to place constraints on the IMF. As a result, they did not explicitly examine the consequences of aging on the form of the HII LF itself. In addition, their assumption of uniform initial cluster masses in the evolutionary model is of only limited use, since actual initial cluster masses apparently have a powerlaw distribution (e.g., Oey \& Clarke 1997; see below). Therefore, we will here investigate the effect of cluster aging on the form of the H II LF itself.

\section{Saturated Population}

We have carried out a preliminary investigation of this problem as part of a different study (Oey \& Clarke 1997), and review the important relevant issues here. A first critical point is the fact that not all clusters have the same relative contribution to ionizing luminosity from the various stellar masses. Such a situation is true for rich clusters that sample the IMF well, but breaks down for sparse clusters in which small-number statistics determine the relative ionizing contribution of different stellar masses. We shall refer to the rich clusters with good stellar statistics as "saturated" with respect to the IMF, and those with poor statistics as "unsaturated." The importance of this fundamental difference in the dominant ionizing contributors between the saturated and unsaturated clusters is demonstrated by a break in the predicted slope of the unevolved H II LF. This change in slope was clearly demonstrated by McKee \& Williams (1997; hereafter MW97), who performed Monte Carlo simulations of clusters drawn from a truncated powerlaw distribution in the numbers of member stars $N_{*}$, and a Scalo (1986) IMF. Since $L \propto N_{*}$ for the saturated objects, the H II LF slope is the same as the parent slope in $N_{*}$; but for unsaturated populations, the H II LF slope is distinctly flatter. This flattening is caused by the increased scatter in the $L$ 's contributed by a bin of given $N_{*}$. The unsaturated condition occurs when this scatter is large compared to the mean
$L$ at the given $N_{*}$, hence the slope of the HII LF flattens. MW97 observed that in their simulations, this change in slope occurs at $L_{\mathrm{sat}}=l_{\mathrm{up}}$, the luminosity contributed by a single star at the upper-mass limit $m_{\text {up }}$, of the IMF. Stellar models currently suggest that $l_{\text {up }}$ is in the range $38.0 \lesssim \log l_{\text {up }} \lesssim 38.5$ (Schaerer \& de Koter 1997; Vacca, Garmany, \& Shull 1996; Panagia 1973). We caution however, that the slope break $L_{\text {sat }}$ in the H II LF does not necessarily occur at $l_{\text {up }}$, as we show in $\S 4.3$.

Investigation of evolutionary effects on the H II LF should therefore consider the differences in stellar ionizing populations between the saturated and unsaturated regime. We examined the behavior of the saturated case analytically in Oey \& Clarke (1997). We considered two extremes in the formation of ionizing clusters: a single-burst scenario, in which all objects are created at the same time; and a continuouscreation scenario. In what follows, we will also assume a constant IMF throughout.

For the case of the single burst, it is apparent that, since all objects follow the same luminosity evolution, the initial slope of the H II LF is simply preserved.

However, in the case of continuous creation, such a conclusion is not obvious. We described the luminosity evolution of the $\mathrm{H}$ II regions in terms of a fading function $f(t)$ (Oey \& Clarke 1997):

$$
L=L_{0} f(t)
$$

where $L_{0}$ is the zero-age nebular luminosity. Stellar population synthesis work (e.g., Leitherer \& Heckman 1995; Beltrametti, Tenorio-Tagle, \& Yorke 1982) suggests that the tail of the fading function $f(t)$ is welldescribed by a power-law in time $t$, so that

$$
f= \begin{cases}1, & t<t_{\mathrm{ms}} \\ \left(t / t_{\mathrm{ms}}\left(m_{\mathrm{up}}\right)\right)^{-\eta}, & t \geq t_{\mathrm{ms}}\left(m_{\mathrm{up}}\right)\end{cases}
$$

For saturated objects, the onset time, $t_{\mathrm{ms}}\left(m_{\mathrm{up}}\right)$, for the fading is the same for all objects. Nebular fading causes initially bright objects to contribute to lower- $L$ bins at later ages, thus if the epoch of fading dominates over the epoch of zero-age luminosity, we would expect the observed HII LF to be steeper than the unevolved one. We found that for $f(t)$ of the form in equation 3 , and reasonable ranges for $m_{\mathrm{up}}, t_{\mathrm{ms}}\left(m_{\mathrm{up}}\right)$, and $\eta$, that essentially no change in H II LF slope is expected. There is an exception for initially shallow 


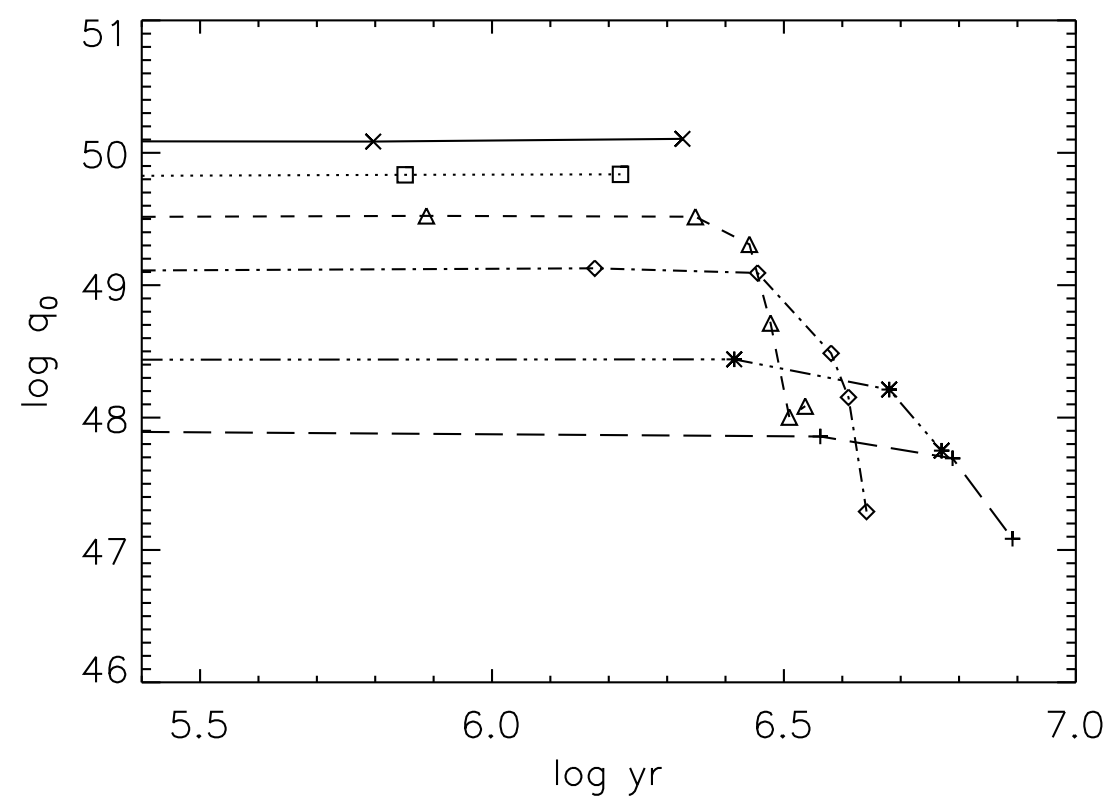

Fig. 1.- Evolution of ionizing photon emission rate $q_{0}\left(\mathrm{sec}^{-1}\right)$ from Schaerer \& de Koter (1997). The stellar models are, from bottom, 20, 25, 40, 60, 85, and $120 \mathrm{M}_{\odot}$.

slopes of $<\eta^{-1}+1$, for which the observed slope indeed steepens to a value $a=\eta^{-1}+1$. This critical value of the slope is probably in the range $1.2-1.4$, which is lower than most measured HII LF slopes, hence it is likely that this effect is unimportant. These effects are described in further detail in Oey \& Clarke (1997). Thus in the saturated regime, no change in H II LF slope is reasonably expected.

\section{Monte Carlo Models}

The statistical uncertainties that dominate the unsaturated clusters render an analytic investigation of these objects unfeasible. This is due primarily to the fact that many different combinations of stellar masses can contribute to any given luminosity, and there is no simple way to relate the total nebular luminosity to the stellar composition, and hence, luminosity evolution. We have therefore modeled the H II LF with Monte Carlo simulations to investigate its behavior in the unsaturated regime.

Our model draws the integer number of ionizing stars from a power-law distribution, so that $N\left(N_{*}\right) d N_{*}$ is the number of clusters with quantities of stars in the range $N_{*}$ to $N_{*}+d N_{*}$ :

$$
N\left(N_{*}\right) d N_{*}=N_{*}^{-\beta} d N_{*} .
$$

We consider "clusters" containing a minimum of one star, with the probability of larger clusters decreasing with $N_{*}$ according to the exponent $\beta$. Our models use the RAN1 portable random number generator described by Press et al. (1986), to generate the random deviate $x$, having values between 0 and 1 . The distribution in $N_{*}$ given by equation 1 is then obtained by

$$
N_{*}=[-x(1-\beta)]^{1 /(1-\beta)},
$$

where $N_{*}$ is assigned to the nearest integer. Since the saturated end of the HII LF does not change slope, as outlined in the previous section, we adopt $\beta=2$ as a typical value found for HII LFs in nearby galaxies (e.g., KEH). Unlike MW97, we do not consider a distribution that is truncated at an upper limit in $N_{*}$ for our default models, although this condition is relaxed in $\S 3.3$.

For each cluster, we then draw $N_{*}$ stars from a stellar mass distribution truncated above a mass limit $m_{\text {up }}$ :

$$
n(m) d m \propto\left[\left(\frac{m}{m_{\mathrm{up}}}\right)^{-\gamma}-1\right] d m
$$

where $n(m) d m$ is the number of stars in the range $m$ to $m+d m$. These are generated with an algorithm analogous to that for $N_{*}$. Equation 6 describes 
a power-law for $m \ll m_{\mathrm{up}}$, and is the same analytic form used by MW97 for their $N_{*}$ distribution and IMF; we refer to this form as a truncated powerlaw, following their nomenclature. We use a Salpeter (1955) power-law slope of $\gamma=2.35$, and adopt an upper-mass limit $m_{\text {up }}=100 \mathrm{M}_{\odot}$. We include stars down to a lower-mass limit $m_{\mathrm{lo}}=17 \mathrm{M}_{\odot}$ (see below). The $\mathrm{H} \alpha$ luminosity $l$ associated with individual stars is dependent on the mass and main-sequence lifetime $t_{\mathrm{ms}}$, for which we also use power law parameterizations:

$$
m \propto l^{\delta},
$$

normalized so that $l_{\text {up }} \equiv l\left(m_{\mathrm{up}}\right)=3 \times 10^{38} \mathrm{erg} \mathrm{s}^{-1}$; and

$$
m \propto t_{\mathrm{ms}}^{-d},
$$

normalized so that $t_{\mathrm{ms}}\left(m_{\mathrm{up}}\right)=2.8 \mathrm{Myr}$ (Schaerer et al. 1993). For stars in the range $17<m<100 \mathrm{M}_{\odot}$, we find a value of $d=0.7$ from Schaerer et al. (1993). We caution the reader that the power-law representations of equations 7 and 8 are approximations; the relations steepen significantly toward lower masses. As discussed below, larger values of $\delta$ imply a greater sensitivity of $l$ to $m$, thereby increasing the range of $L$ in the unsaturated regime, for a given range of $m$. We adopt $\delta \sim 1.5$, which yields a reasonable $\log l_{\mathrm{lo}} \sim 37.3$ for $m_{\mathrm{lo}}=17 \mathrm{M}_{\odot}$. For illustrative purposes, it is helpful to show a larger dynamic range of unsaturated objects, though we caution that in reality the range of these objects is likely to be somewhat smaller for our mass limits, since the $m-l$ relation flattens toward higher masses (e.g., Leitherer 1990). We have adopted the value of $m_{\text {lo }}$ to coincide roughly with the value at which the $l-m$ relation turns over more steeply, since stars below $\sim 17 \mathrm{M}_{\odot}$ do not contribute strongly to the ionizing fluxes. There is also a relative increase in $t_{\mathrm{ms}}(m)$ at lower masses, but not as significant as the change in $l(m)$. It is useful to bear in mind that the representative values of $\delta$ and $d$ will increase toward lower masses.

We consider two different formulations for the stellar ionizing rate $q_{0}$ with time. The first simply approximates that $q_{0}$ remains constant until $t_{\mathrm{ms}}$ and is zero thereafter. The second formulation accounts for evolution of $q_{0}$ during the stellar main-sequence phase. Figure 1 shows $q_{0}$ for stars of different initial masses as a function of time, from the models of Schaerer \& de Koter (1997). These models suggest that $l$ remains fairly constant until a time after which it decreases roughly along the relation,

$$
l=l_{\mathrm{up}}\left(\frac{t}{t_{\mathrm{ms}}\left(m_{\mathrm{up}}\right)}\right)^{-\zeta},
$$

where $\zeta \sim 5$, and $t$ is the age from birth. Given the uncertainties in massive star evolution and ionizing fluxes, we adopt the crude approximation of constant $l$ until the time of intersection with equation 9, after which we adopt $l$ from that relation. One consequence of this formulation is that stars with initially different luminosities can have the same $l$ during certain of their fading periods. Figure 11 suggests that this may not be far from reality. We caution that the assumption of coeval star formation within individual nebulae is likely to be an oversimplification, and that the stellar age spread is typically similar to the main-sequence lifetime of the most massive stars (e.g., Massey et al. 1995). Therefore, the early nebular luminosity evolution could potentially increase somewhat before the onset of fading. Specific modeling of star formation scenarios would be necessary to investigate this effect.

Our adopted lower-mass cutoff introduces artificial fluctuations that can be seen in all our models at low $L$. The quantized nature of the stellar ionizing sources, combined with the truncation of stellar masses below $m_{\mathrm{lo}}$, causes artificial kinks in the distribution of nebular luminosities at low $L$. For example, the most prominent of these features appears at $\log L=37.6$ in our models, or 0.3 dex above the lower- $L$ cutoff of $\log l_{\mathrm{lo}}=37.3$. This dip results from the fact that all nebulae at $L \lesssim 37.6$ contain single stars, selected according to the IMF, whereas two-star nebulae begin to contribute above this luminosity. To more clearly demonstrate the features in the H II LF, we therefore include $10^{4}$ clusters in our simulations, although this number is higher than is seen in most nearby galaxies. Finally, we truncate our model H II LFs below the minimum luminosity $l_{\mathrm{lo}}$, which is that due to a single star of mass $m_{\text {lo }}$. Although objects originating at higher $L$ evolve to luminosities below $l_{\text {lo }}$ for cases incorporating main-sequence evolution, the omission of objects originating below $l_{\text {lo }}$ renders the model populations incomplete below that value.

\subsection{Single Burst}

Figure 2 shows two models for the evolution of an $\mathrm{H}$ II region population created in a single burst, with $\delta=1.5, d=0.7$, and $17<m<100 \mathrm{M}_{\odot}$. The left 
column of Figure 2 (panels $a-c$ ) shows model A1, with constant $l$ during the main-sequence; while the right column (panels $d-f$ ) shows model A2, with $l(t)$ as described above, to include main-sequence fading. The zero-age H II LFs are given in the top panels, with the middle and bottom panels showing ages of 4 and 7 Myr, respectively. Note that the two zero-age models (top panels) are statistically identical. To aid comparison of the models, the dotted line corresponding to $L_{\text {sat }}=3 \times 10^{38} \mathrm{erg} \mathrm{s}^{-1}$ is shown for reference.

As found in the simulations of MW97, the zeroage models in Figure 2 (top panels) show a flattening of the HII LF slope below a saturation luminosity. For the adopted stellar parameters, this transition from saturated to unsaturated populations occurs at $\log L_{\mathrm{sat}}=\log l_{\mathrm{up}}=38.5$. Equation 7 shows that the extent of this unsaturated regime is determined by $\delta$ : a larger $\delta$ implies a greater sensitivity of $l$ to $m$, thereby increasing the range in $L$ of this population, for a given range of $m$. This effect also causes the slope in the unsaturated regime $\left(L<L_{\text {sat }}\right)$ to be dependent on $\delta$ because the scatter in $L$ contributed by individual stars is larger for large $\delta$, and this scatter determines the flattening in slope. Hence, larger values of $\delta$ will induce a flatter slope. As mentioned above, our adopted value of $\delta=1.5$ is an overestimate for the more massive stars in the IMF, hence the unsaturated slope should be somewhat steeper near $L_{\text {sat }}$ than is seen in our models.

H II regions with $L>L_{\text {sat }}$ are not sensitive to the $m-l$ relation since statistical effects are unimportant in this regime. The slope of the HII LF in the saturated regime is $\beta$, the same as that of the parent

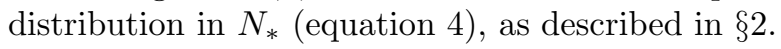

Figures $2 b$ and $c$ show Model A1 at ages of 4 and 7 Myr respectively. These ages should not be regarded too literally, since the evolutionary state at a given age is dependent on equation 8, which is an approximate parameterization, as well as equations 6 and 7 . For reference, $t_{\mathrm{ms}}\left(m_{\mathrm{lo}}\right)=9.7 \mathrm{Myr}$ for our adopted $d=0.7$. It is immediately apparent that the inflection in slope moves toward progressively lower luminosities with age. This is primarily a reflection of the group evolution of the saturated population to lower luminosities; the entire distribution at $L>L_{\text {sat }}$ in Figure $2 a$ is moving toward lower luminosities in Figures $2 b$ and $c$. For cases such as this, in which the zero-age $L_{\mathrm{sat}}=l_{\mathrm{up}}$, the slope inflection at later ages now corresponds to $l$ of the most massive star still present in these objects. More generally, the rate at which the saturated objects fade in $L$ has a dependence of $t^{\delta / d}$, as determined by equations 7 and 8 .

Model A2 includes main-sequence evolution of the ionizing fluxes. We caution that the models including the main-sequence evolution should be interpreted qualitatively, owing to the crude parameterization of the stellar evolution. The principal feature is that Model A2 clearly evolves much faster than Model A1. The other important effect is that noticeable, sharp structure in the HII LF becomes apparent near the transition from saturated to unsaturated objects, as seen in Figure 2e as contrasted to Figure 2 $b$. This results from the fact that clusters dominated by a few, high-mass stars are now capable of evolving to lower $L$ than clusters dominated by many, unevolved, lowmass stars. In other words, stellar evolution allows the low- $L$ end of the saturated population to overtake, toward lower luminosities, the high- $L$ end of the unsaturated population. The details of this structure in the HII LF are dependent on the details of the main-sequence luminosity evolution.

\subsection{Continuous Creation}

Figure 3 shows simulations for a population where the $\mathrm{H}$ II regions are being continuously created (Model B1). This model is the same as that in the single burst case with no main-sequence luminosity evolution (Model A1), but now with each new cluster formed at a uniform incremental time after its predecessor. The total distribution of initial luminosities, $\log L_{0}$, of all the objects, which represents the parent, unevolved $\mathrm{HII} \mathrm{LF}$, is the same as the zero-age distributions in Models A1 and A2 (Figures 2 $2 a$ and $d$ ). Figure $3 a$ shows the distribution at an age of $6 \mathrm{Myr}$ after the onset of continuous creation, but before the population has achieved its steady-state form. The steady-state H II LF, which occurs at $t>t_{\mathrm{ms}}\left(m_{\mathrm{lo}}\right)$, is shown in the Figure $3 b$.

We may consider the HII LF for continous creation to be a sum of individual bursts with a continuous age distribution. Since the single burst H II LF evolves predominantly as a progression of the saturated objects, with slope $\beta$, to the lower luminosities of the unsaturated regime, we therefore expect that the slope in the region $L<L_{\text {sat }}$ will be intermediate between its flatter value in the unevolved case and $\beta$. Figure $3 b$ shows that this is indeed the case. We can also see in Figure $3 a$, that the intermediate evolutionary stage shows the region of steepening moving in from higher luminosities, since the low-luminosity 

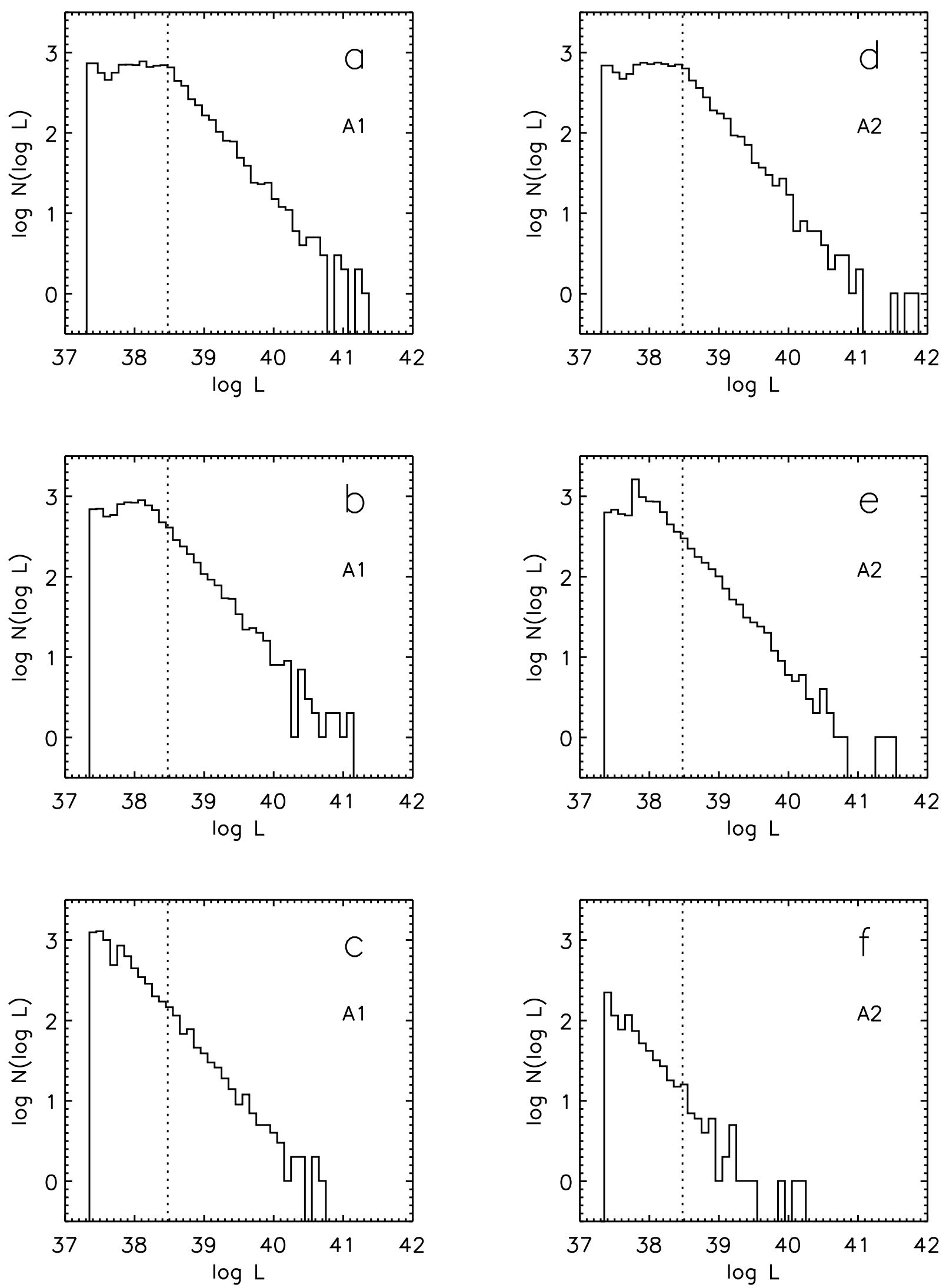

Fig. 2.- Model H II LFs for a single burst. Model A1 (panels $a-c$ ) considers constant $l$ over main sequence lifetimes; Model A2 (panels $d-f$ ) considers main sequence fading of $l$ as described in $\S 3$. The dotted line marks $L_{\mathrm{sat}}=3 \times 10^{38} \mathrm{erg} \mathrm{s}^{-1}$. Ages of 0,4 , and $7 \mathrm{Myr}$ are 7 shown from top to bottom, respectively. The dip near $\log L \sim 37.6$ is a quantization artifact induced by the lower-mass cutoff. [Note that the slope of $\log N(\log L)$ vs. $\log L$ is $1-\beta$.] 

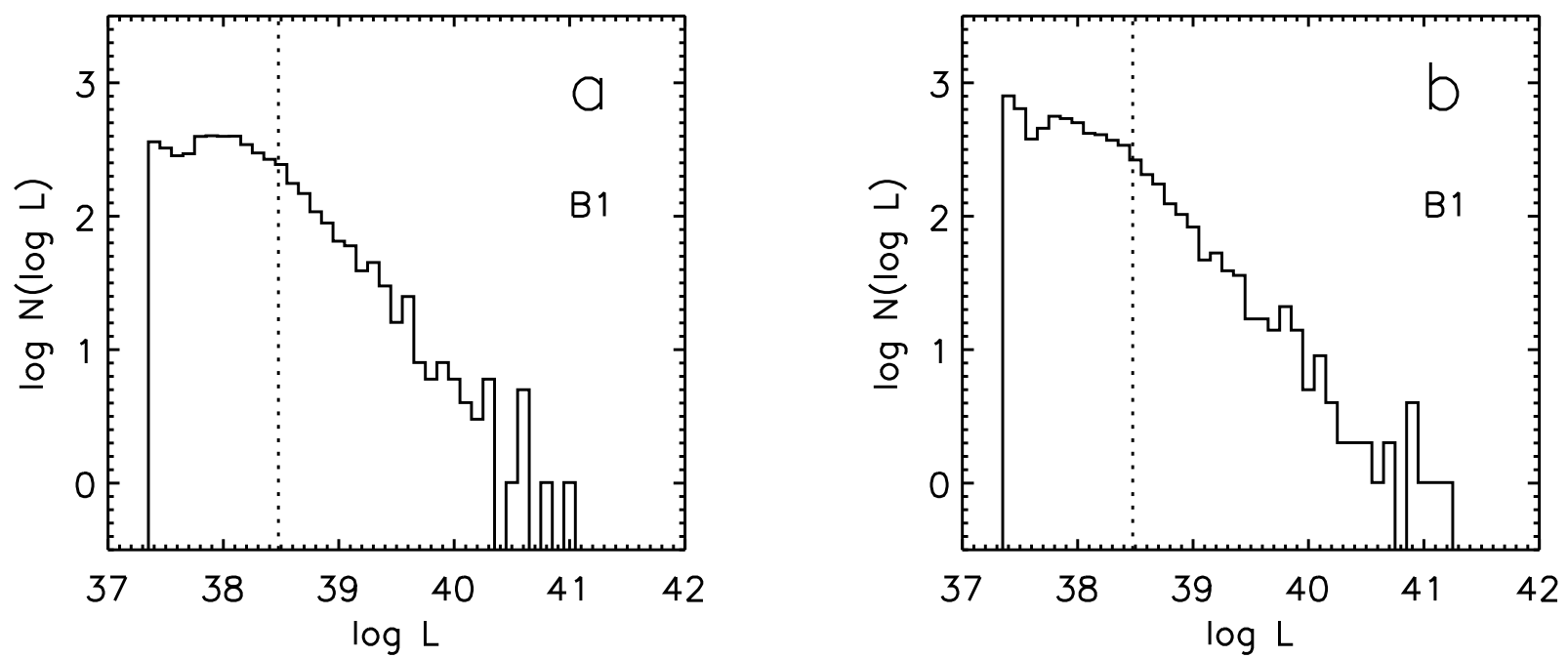

Fig. 3.- H II LF Model B1, for continuous creation, with constant $l$ over main sequence lifetimes. Panel $a$ shows an age of $6 \mathrm{Myr}$ after the onset of continuous creation, before steady-state is achieved. Panel $b$ shows the steady-state form. (The dip near $\log L \sim 37.6$ is the same quantization artifact seen in Figure 2.)

bins are not yet affected by saturated, evolved objects. We also generated a Model B2, incorporating main sequence luminosity evolution, analogous to Model A2. The steady-state form of that model also shows an intermediate-value slope at $L<L_{\text {sat }}$, but slightly flatter than its analog in Model B1 because of the structure present at $L<L_{\mathrm{sat}}$ in the burst case (Model A2).

\subsection{Unsaturated Population}

In Figure 4, we show models for a population of only unsaturated clusters. These are drawn from the same power law distribution in $N_{*}$ (equation 4 ) as the previous models, but only for $N_{*} \leq 10$. For our default stellar parameters with $17<m<100 \mathrm{M}_{\odot}$, the saturation transition in $N_{*}$ is roughly between 10 and 20 stars. We use the same stellar parameters as in the previous models, now with a total of 3000 clusters. The left side of Figure 1 shows the simulation for the unevolved population $(a)$, and continuous creation (b); while the right side shows the single burst case at ages $4 \mathrm{Myr}(c)$ and $7 \mathrm{Myr}(d)$. These models do not include main sequence luminosity evolution, whose consequences are analogous to those discussed for Models A2 and B2 above.

It is apparent in Figure 1 that the primary difference in the HII LF between this population and the complete power-law sampling in $N_{*}$, is that the high- $L$ tail of the unsaturated objects falls off very steeply. The highest-luminosity, unsaturated clusters result from a chance assemblage containing a disproportionate number of high-mass stars. Therefore, the distribution of these objects must fall more steeply than that of a saturated population, owing largely to the scarcity of the high-mass stars. The actual slope in this regime is once again determined by the detailed statistics of the cluster compositions, and equations 6 and 7 .

At $L<L_{\text {sat }}$, the behavior of these unsaturated populations is identical to that seen in the models with a full distribution in $N_{*}$, since, as discussed above, this range in $L$ is dominated by the unsaturated objects. It is only at the high- $L$ limit itself, in the evolved case of Figure $4 d$, that the sudden drop due to sheer lack of objects, effectively generates a steep slope. Note that at luminosities below this high$L$ drop-off, the H II LF in this model recovers a slope of $\beta$, analogous to Figure $2 c$.

\section{Observed H II LFs}

The features and behavior found in the simulated H II LFs can provide a useful interpretation of the patterns found in observed HII LFs.

\subsection{Arm and Interarm $\mathrm{H}$ II LFs}

In Figure 5, we reproduce the published H II LFs for arm and interarm populations in six grand de- 

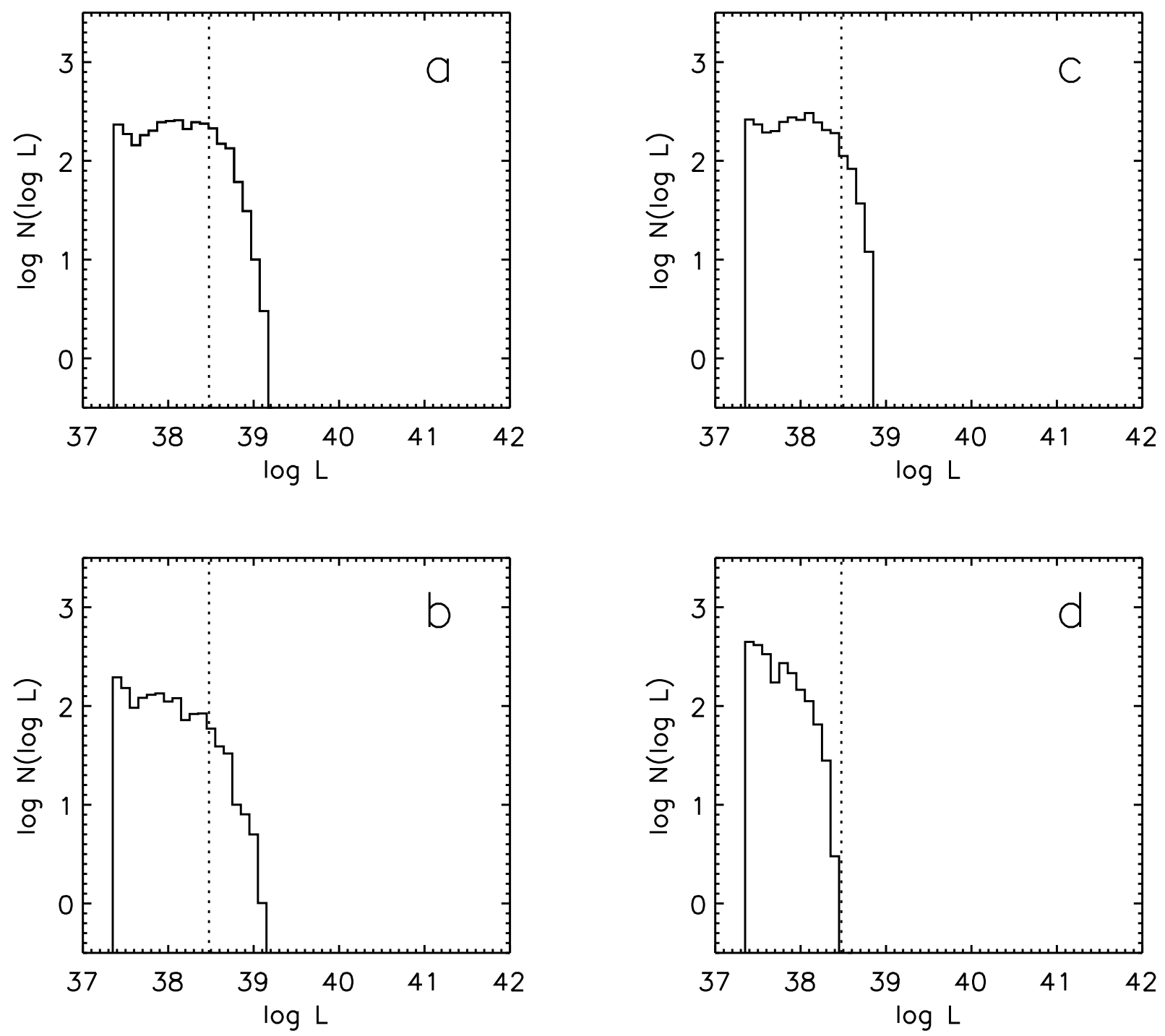

Fig. 4.- Models with the same parameters as in Figures 2 and 3 , but for populations with $N_{*} \leq 10$. (a) Unevolved population; (b) steady-state, continuous creation; $(c)$ single burst at age $4 \mathrm{Myr}$; $(d)$ single burst at age $7 \mathrm{Myr}$. To aid comparison, these models are plotted on the same scale as Figures 2 and 3 . 
sign spirals. The solid histograms show the arm H II LFs, and the dot-dashed lines show the interarm H II LFs. In each case, the peak of the interarm H II LF occurs at a fainter luminosity than that for the arm regions, suggesting that the interarm H II LF mirrors that of the arms at lower $L$. This shift in peak occurs despite the smaller total numbers of $\mathrm{H}$ II regions in the interarm areas. Owing to incompleteness effects, we might otherwise expect the smaller numbers to induce a turnover at, if anything, higher $L$. We do caution that blending of nebulae (Deharveng et al. 1988; KEH) in the more crowded arm populations could also introduce a flattening in the observed H II $\mathrm{LF}$ at lower $L$. However, as pointed out previously (Rand 1992; Knapen et al. 1993; KEH), the highest$L$ objects in the interarm regions are apparently always fainter than those in the arms. This is again consistent with a group shift in luminosity.

These features exhibit the behavior of our model H II LFs for the burst scenario. This is consistent with the expectation that the arm nebulae are largely coeval, and are undergoing a current burst of cluster formation; whereas the interarm regions represent, on average, an older population remaining from previous star formation. As seen in Figure 2, the inflection in the HII LF occurs at progressively lower values with age. Note that even if incompleteness affects the location of the peak for the arm objects, which we take to reflect the unevolved population, the relative shift in the peak for the interarm objects is still consistent with evolution, owing to the steeper slope at lower $L$. This is because the low- $L$ bins are more robust to incompleteness for a steep slope than for a shallow one, since the proportion of missing objects is the same in each case. Therefore, while the absolute location, or existence, of the H II LF peak may be suspect due to observational limitations, the observed shift in peak for the interarm regions is fully consistent with our model because a steeper slope is expected at low $L$ (cf. Figure 2 $2 d$ and $f$ ). Indeed, given that the H II LF peak is often substantially lower in $\log N(\log L)$ for the interarm regions, this suggests that the actual peak of the distribution has moved to luminosities much lower than the empirical completeness limit, as is illustrated, for example, in Figure $2 f$.

The aging effect can also explain the steeper H II LF slopes that are sometimes reported for the interarm regions as compared to the arm regions of spiral galaxies (Point 2 in $\S 1$ ). If the actual nebular luminosity function conforms to the two-slope shape seen in the models, and if the two slopes are not fitted separately, then the net effect is that the measured slope will be intermediate between the two. In our single burst model, the location of the break moves to lower $L$. Therefore, over a fixed range of $L$, the contribution of the shallower slope to the composite, measured H II LF slope will be less for an evolved burst population, than for an unevolved one. As is apparent in Figure 5, it is difficult to pinpoint the location of the slope change. We mention once again that our simulations show a flatter slope in the unsaturated regime than would be expected, owing to the parameterization of equation 7 . Furthermore, the observed location of $L_{\text {sat }}$ will also vary with galactic internal extinction, which most H II LFs, including the ones in Figure 5, do not take into account. With these difficulties, we suggest that the slopes of the interarm regions do appear to be the same as those in the saturated regime of the arm populations.

Thus the differences observed in the HII LFs for arm and interarm regions are not necessarily due to changes in star formation environment, but are likely to be affected by evolution. The time scale between the passage of spiral density waves is of order $\sim 40$ Myr (e.g., Rand 1993) at any given location in the disk, which is much longer than the observable life expectancy of the H II regions. Thus, the spatial and luminosity distribution of nebulae between the arms does imply that there is at least a certain amount of bona fide interarm star formation taking place. However, based on the arguments presented in $\S 1$, we feel that an aging population is likely to be an important component in the interarm regions, and that the behavior of the HII LF is consistent with this interpretation. With the present data and the observational uncertainties mentioned above, combined with uncertainties in predicted $q_{0}$ and its evolution, we have not attempted to place meaningful age constraints on the evolved population. However, it will be interesting to re-examine the data more closely, and search for an evolutionary progression in the H II LF with distance behind the leading edge of the spiral arms. We hope to carry out such an investigation in the near future.

\subsection{The Hubble Sequence}

The correlation of earlier type galaxies with steeper slopes (Point 3 in $\S 1$ ) can be explained by systematically lower cutoffs in $N_{*}$ in those clusters. KEH have shown compelling evidence that these galaxies have fewer stars per cluster, since the mean nebular lu- 

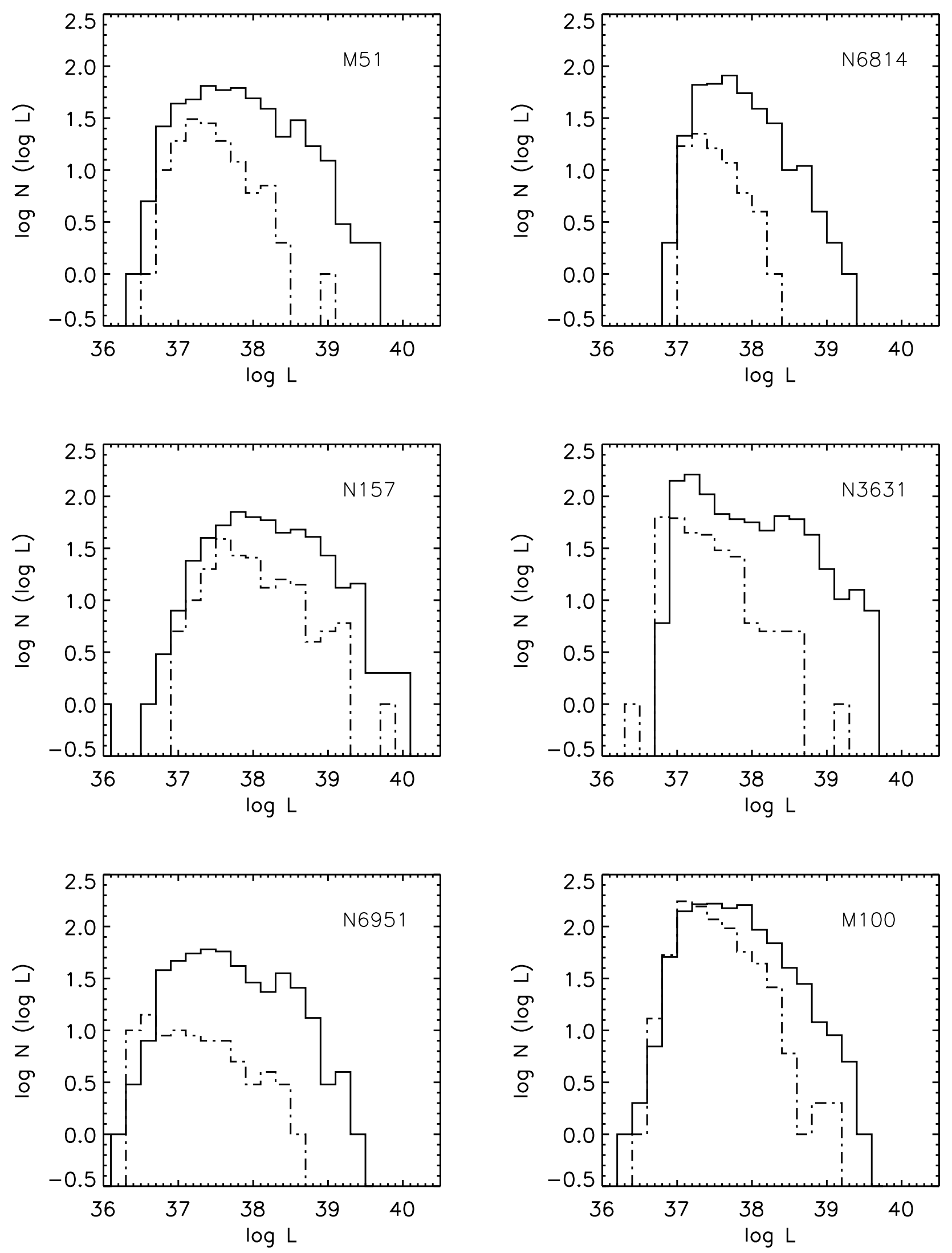

Fig. 5.- Observed H II LFs for arm (solid lines) and interarm (dot-dashed lines) populations in grand design spirals: M51 (Rand 1992); NGC 6814 (Knapen et al. 1993); NGC 157, NGC 3631, and NGC 6951 (Rozas et al. 1996a); M100 (Knapen 1997). 
minosities in the early type spirals are substantially lower than those in late types, despite the larger total numbers of $\mathrm{H}$ II regions in the earlier types. Therefore, unless the stellar ionizing population varies drastically, it is probable that the size of the parent clusters must themselves be smaller on average in the earlier type galaxies. The same conclusion has been found for Sa galaxies (Caldwell et al. 1991), although the total numbers of $\mathrm{H}$ II regions are again smaller in those galaxies.

The decrease in typical $N_{*}$ per cluster with Hubble type (KEH) could easily result from a decrease in the upper cutoff of the $N_{*}$ power-law. The earliest type galaxies would have populations composed only of unsaturated objects, as would correspond to Sa galaxies, where the maximum $\log L$ is typically in the range $38.0-38.5$ (Caldwell et al. 1991). This is the same range in values as the estimates for $l_{\mathrm{up}}$, as mentioned earlier, hence it is indeed the regime of unsaturated objects. As seen in $\S 3.3$, the models that describe this population show a steeper high-luminosity tail above $L_{\text {sat }}$, than do the populations with saturated objects. Indeed, the slopes in Sa galaxies have been found to be even steeper than those for $\mathrm{Sb}$ - Sc types: the typical H II LF slope $a \sim 2.6$ for Sa galaxies (Caldwell et al. 1991), as compared to $a \sim 2.0$ for $\mathrm{Sb}-\mathrm{Sc}$ galaxies (KEH; Banfi et al. 1993). The model in Figure $\$ b$, for continuous creation of unsaturated objects, would therefore be the most appropriate of our simulations to describe the Sa H II LFs. The form of the Sa H II LFs shown in Figure 5 of Caldwell et al. (1991) show a consistent resemblance with our models in Figure 1 . Thus, ironically, although these nebulae are dominated by unsaturated objects whose characteristic slope is flatter than the saturated case, the high- $L$ drop-off will induce a steeper measured slope in these populations.

The $\mathrm{Sb}$ - Sc galaxies would then show a slope intermediate between the Sa and Im types (cf. Figures $4 b$ and $3 b$ ), as the upper cutoff in $N_{*}$ approaches the exclusively unsaturated regime. Indeed, KEH suggest that the H II LF in $\mathrm{Sb}-\mathrm{Sc}$ galaxies is truncated at an upper- $L$ limit. This is quantitatively supported by MW97, who also find evidence for a truncated H II LF in the Milky Way and Sb-Sc galaxies, but not in irregular galaxies. Since this limit is still at $L$ above the regime of unsaturated objects, these findings are consistent with our interpretation of a progressively lower upper- $N_{*}$ cutoff with earlier Hubble type. There may be exceptions caused by temporarily enhanced star formation, in which large numbers of saturated clusters are formed. This scenario can explain the larger range in H II LF slope seen in earlier type galaxies (KEH). Indeed, a slope steepening at high $L$ reported in some earlier type galaxies could well correspond to a cutoff; indeed, KEH note that it is often impossible to distinguish such a turnover from a cutoff. Thus the steepening in slope for disk galaxies across the Hubble sequence can be explained by a simple trend in upper cutoff of the $N_{*}$ distribution, and need not invoke a change in the parent slope $\beta$ of $N_{*}$.

\subsection{The Slope Transition}

In $\S 3$, we denoted the luminosity of the slope break in the initial H II LF by $L_{\text {sat }}$ and noted that, as in the models of MW97, this often corresponds to the luminosity contributed by most luminous star in the $\mathrm{IMF}, l_{\mathrm{up}}$. We however caution that this correspondence is dependent on the stellar parameters. As an example, Figure 6 exhibits the H II LF resulting from two models with identical IMF slope and $m-l$ relation, but with different stellar mass limits. Figure 6 a, with $3<m<100 \mathrm{M}_{\odot}$, exhibits an H II LF that more closely resembles in form the simulation by MW97. There are two principal slope breaks apparent: one near $l_{\mathrm{up}}$; and one near $\bar{l}$, below which the slope appears quite flat. The existence of a minimum cluster membership number, $N_{*}=1$, causes this flattening, or in some cases even positive slope, for luminosities below the expectation value of $L$ containing single stars, i.e., the average stellar luminosity $\bar{l}$. At $\bar{l}<L<l_{\text {up }}$, there is an intermediate slope in the HII LF that joins the flat regime to the saturated regime of slope $\beta$. A small transition region of $\lesssim 0.3$ dex is noticeable in this intermediate-slope region near both $\bar{l}$ and $l_{\text {up }}$, which results from the form of the IMF adopted in equation 6. With instead a sharp IMF truncation at $m_{\text {up }}$, the slope breaks would occur cleanly at $\bar{l}$ and $l_{\text {up }}$.

One can now see that certain stellar mass ranges and values of $\delta$ can change the relative location of the two breaks such that one or the other could disappear. The model in Figure $6 b$, with $17<m<$ $300 \mathrm{M}_{\odot}$, shows an example where the $l_{\text {up }}$ slope break is virtually undetectable at $\log l_{\text {up }}=39.2$. The smaller difference between $\bar{l}$ and $l_{\text {up }}$ has compressed the intermediate-slope range in $L$-space, thereby steepening its slope to a value closer to $\beta$, which represents the maximum slope possible in this regime. An observed H II LF similar to Figure $6 b$ would most likely 

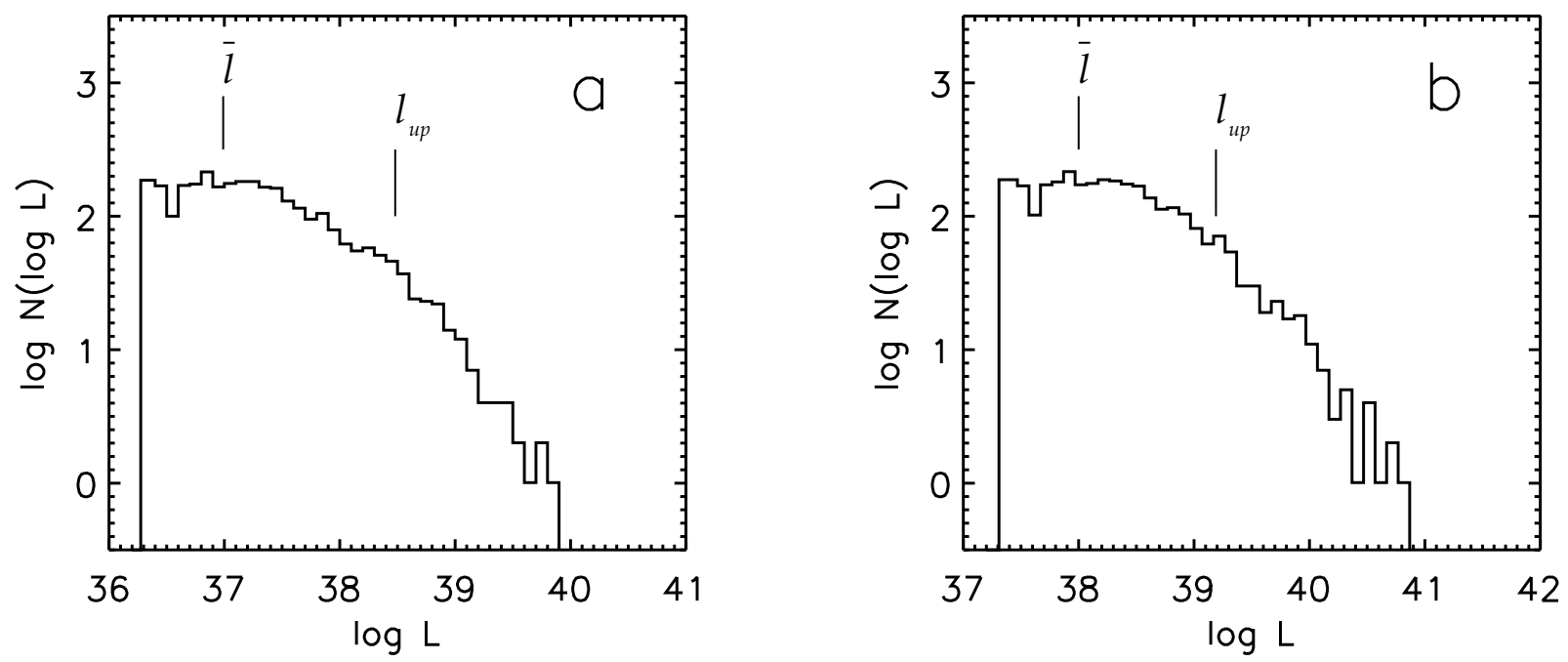

Fig. 6.- (a) Model for $3<m<100 \mathrm{M}_{\odot}$; and $(b)$ model for $17<m<300 \mathrm{M}_{\odot}$. The location of $\bar{l}$ and $l_{\text {up }}$ are indicated in each case.

be measured to have only one slope in the regime $L>\bar{l}$. Note that the models in Figure 6 are probably unrealistic, since $\delta=1.5$ is not representative over such large mass ranges, and we only show these for illustrative purposes. However, these examples demonstrate that breaks in observed H II LFs should be interpreted with caution, and may only provide a lower limit to the most luminous star in the IMF. Furthermore, the tendency of the highest-mass stars to approach similar ionizing emission rates $q_{0}$ (e.g., Leitherer 1990), compounds the insensitivity of $l_{\text {up }}$ to $m_{\text {up }}$. We therefore conclude that, unfortunately, the H II LF is not a useful probe of $m_{\mathrm{up}}$.

However, the insensitivity of $l_{\text {up }}$ to $m_{\text {up }}$ suggests that the high- $L$ end of the H II LF should be a reliable indicator of $\beta$ for saturated nebular populations, since it diminishes the likelihood of a varying $m_{\mathrm{up}}$ as an explanation for the observed variations in the H II LF. The principal caveat is that a truncation at maximum $N_{*}$ not be confused with a steep slope of $\beta$, as may have been done for Sa galaxies in the past (see above). It is also apparent that, since the form of the H II LF is sensitive to the stellar parameters, that demonstrable variations in the unsaturated regime of the zero-age or continuous creation H II LF could possibly indicate substantial variations in the stellar parameters of the hottest stars.

We note that stochastic variations in $m_{\text {up }}$ within a galaxy will not change the location of the break if the distribution of $m_{\mathrm{up}}$ is gaussian. We confirmed this by drawing $m_{\text {up }}$ from a parent normal distribution of $\sigma=15 \mathrm{M}_{\odot}$ and the same mean value of $100 \mathrm{M}_{\odot}$. The resulting $\mathrm{HII} \mathrm{LF}$ is indistinguishable from that with a constant $m_{\text {up }}$.

Empirically, a flattening at lower $L$ is often seen in many galaxies (Point 1 in $\S 1$ ), but at varying critical luminosities. While the models for a single burst show that the transition point is not necessarily fixed, $L_{\text {sat }}$ cannot be greater than $l_{\text {up }}$. At present, the value of $l_{\mathrm{up}}$, the $\mathrm{H} \alpha$ luminosity associated with $m_{\mathrm{up}}$, is not well-determined. Our models have adopted a value of $l_{\mathrm{up}}=3 \times 10^{38} \mathrm{erg} \mathrm{s}^{-1}$, which is uncertain within a factor of a few, and is a high estimate. Most published H II LFs are not corrected for internal extinction within the galaxies, which will affect the location of the break. As can be seen in Figure $8 b$ of Walterbos \& Braun (1992), extinction correction for the highlyinclined case of M31 can shift the location of the apparent turnover by an order of magnitude. It is also important to distinguish between a real slope change and artificial slope flattening induced by limitations such as incompleteness, and the more insidious problem of object blending, mentioned above, for more distant galaxies. In view of the difficulty in pinpointing the location of a slope change, and the variations induced by reddening and possible aging effects, we suggest that the published H II LF slopes are thus far consistent with our models.

We note that KEH modeled the lower- $L$ end of the H II LF by combining the $m-l$ relation with the 
IMF for single-star H II regions. This representation is somewhat different from ours, since we include multiple-star, low- $L$ objects that will contribute to the same luminosity bins occupied by bright, singlestar nebulae. Our slope in this regime will therefore tend to be slightly flatter (more positive) than theirs. This will affect estimates for the fraction of total galactic ionizing emission due to faint $\mathrm{H}$ II regions below empirical completeness limits, such that those using the KEH model are an upper limit for similar stellar parameters.

Additional models of the HII LF by Feinstein (1997) came to our attention during the refereeing process. These models consider only nebular populations with luminosities scaled from objects with a saturated IMF, but with $m_{\text {lo }}$ extending down to $5 \mathrm{M}_{\odot}$. We emphasize that, since unsaturated clusters are not considered, the slope turnover seen in those models is therefore not caused by the same mechanism seen in our work, or that of KEH and MW97. Rather, the slope break seen in Feinstein's models result from the adopted lower cutoff in the $L$ distribution, combined with an extended creation period. The zero-age, saturated H II LF is described by the power-law $\beta$, which simply shifts to lower luminosity with advancing age, as exhibited by the saturated tail of our models in Figure 2. Feinstein's continuous creation models are therefore a superposition of many power-laws with a continuous range of ages. The lower- $L$ cutoff seen in those models therefore induces a flattening in the H II LF below that luminosity. This is best illustrated in the right panel of Feinstein's Figure 3, which varies the lower- $L$ cutoff according to assumed total stellar mass, for continuous creation. The slope break occurs at the lower- $L$ cutoff, hence, as pointed out by the author, this parameter determines the location of the break. We caution, however, that the lower- $L$ cutoffs used in Feinstein's models are necessarily artificial, owing to the consideration of only saturated H II regions. Therefore, the slope breaks seen in these models must be regarded as artificial to the same extent. If, however, nebular creation scenarios exist with such a strong lower- $L$ cutoff, Feinstein's models demonstrate another mechanism which can also induce a slope break.

Previous work has sometimes suggested an empirical slope change at high luminosities of $\log L \sim 38.6$ or even higher (Rozas et al. 1996a; Rand 1992; KEH). If a break at these high luminosities is real, it is unlikely to result from the stochastic effect below $L_{\text {sat }}$. How- ever, if the high- $L$ slope is steep enough, it could well be a manifestation of the maximum $N_{*}$ cutoff in those galaxies, which is expected to vary in the scenario we presented in $\$ 4.2$. It could also result from a change in $L_{\text {sat }}$, although this appears unlikely, as argued above. But factors other than those that we have considered can affect the form of the H II LF as well. For example, Rozas et al. (1996a,b) suggest that such a break at high $L$ results from a transition between radiationbounded nebulae at fainter luminosities, to densitybounded objects at brighter luminosities. Another potentially important effect is a differential extinction pattern among the nebular population, and of course, the usual differences in IMF slope and star forming conditions. Given the currently available data, the systematic presence of an additional, high-luminosity slope change at a fixed $L$ remains controversial.

\section{Conclusion}

We have modeled the H II LF with Monte Carlo simulations that draw $N_{*}$ for each cluster from a power-law distribution of constant slope, and for each cluster, $m$ from a Salpeter IMF truncated at $m_{\mathrm{up}}=$ $100 \mathrm{M}_{\odot}$. As found by MW97, this produces shallower slopes corresponding to the population dominated by unsaturated objects, and a steeper, high- $L$ side dominated by saturated ones. The saturated slope is $\beta$, the slope of the $N_{*}$ distribution (equation 4 ). The transition luminosity $L_{\text {sat }}$ in the unevolved H II LF may correspond to $l_{\text {up }}$, the $\mathrm{H} \alpha$ luminosity due to a single star of mass $m_{\mathrm{up}}$, but this depends on the assumed stellar parameters. The observed H II LFs do often show flatter slopes at lower luminosities that are consistent with the transition at $L_{\text {sat }}$ (e.g., Walterbos \& Braun 1992; Scowen, Dufour, \& Hester 1992).

We have hitherto not discussed the form of the H II LF below $l_{\text {lo }}$, which is unexplored in our models. Since the slope $\delta$ of the $m-l$ relation (equation 7) steepens, the H II LF will continue to grow flatter for the same $N_{*}$ power law, extending to lower luminosities. At $\log L \lesssim 36$, contamination from planetary nebulae may become important (Walterbos \& Braun 1992), while confusion and background diffuse $\mathrm{H} \alpha$ emission will undoubtedly limit the H II LF in this regime.

Our simulations show that for a single-burst case, the slope transition moves to lower $L$ as the population ages and the saturated objects grow fainter (Figure 2). Hence, the steady-state model for continuous creation shows a slope in the region $L<L_{\text {sat }}$ that is 
intermediate between its value in the unevolved H II LF and the slope $\beta$ of the saturated objects (Figure 3). Models that include main-sequence evolution for stellar ionizing luminosities show a faster evolution than those that do not, with a noticeable peak around the transition between saturated and unsaturated populations (Figure 2e). The steady-state model for continuous creation incorporating stellar evolution yields a slightly shallower slope than that for the case without stellar evolution, but still steeper than the unevolved case. Models for a population of only unsaturated clusters with $N_{*} \leq 10$ show a much steeper drop-off at $L>L_{\text {sat }}$ (Figure 4 ), where the H II LF is normally dominated by saturated objects. The behavior at $L<L_{\text {sat }}$ is generally the same as that for the models with fully populated $N_{*}$, with the exception of a high- $L$ drop-off in an evolving burst.

The patterns seen in observed H II LFs in nearby galaxies can be explained by the behavior of these models. If H II regions in spiral arms of galaxies represent a current burst of coeval star formation, then the steeper slopes seen in interarm vs. arm regions can be explained as an aging burst compared to a current burst population. Since single slope measurements will yield a composite of the saturated and unsaturated slopes, the zero-age H II LF will show a flatter slope owing to the larger range of unsaturated objects. The lower peak $L$ and maximum $L$ seen in the interarm regions are also consistent with a dominant contribution from an aging population.

The steeper slopes seen in earlier galactic Hubble types can be explained by the lower cutoff in maximum $N_{*}$. As seen in the models for populations of only unsaturated clusters (Figure \#), the high- $L$ tail is much steeper than in populations with higher $N_{*}$. A truncation at the high end of the H II LF has been suggested by KEH and MW97. Since the mean $N_{*}$ does apparently decrease in earlier type galaxies to the values in unsaturated clusters (KEH; Caldwell et al. 1991), the steeper H II LF slopes are likely to simply result from a lower maximum $N_{*}$. A slope dropoff therefore may also be seen at $L$ corresponding to the maximum $N_{*}$.

Thus, we suggest that the current data are consistent with evolutionary effects and the upper- $N_{*}$ cutoff being the primary determinants of the shape of the H II LF. Indeed, it may be unnecessary to invoke any other parameters for the nebular and cluster properties to explain the behavior of the HII LF as described in $\S 1$. If this is true, it is rather remarkable, for example, that grand design spirals with strong star formation induced by tidal interactions, would show the same power-law distribution in $N_{*}$ as those found in normal disk and irregular galaxies. However, we again acknowledge that other, unexplored effects, such as gas density bounding, differential reddening, and variations in IMF and other stellar parameters, can influence the form of the H II LF. Nor can we rule out suggested environmental influence like variations in slope of the molecular cloud mass spectrum. But thus far, it appears that the power-law slope $\beta$ of $N_{*}$ might vary even less than was suggested by the observed slopes of the H II LF, even though the rate and total star formation can vary by one to two orders of magnitude. Further detailed observations of the H II $\mathrm{LF}$, and re-examination of those in the literature, are of great interest in evaluating the possibility of a universal $\beta \sim 2$. Suggestively, Caldwell et al. (1991) find that the luminosity function of knots seen in the $B$ continuum of four of their Sa galaxies do show such a power-law exponent $\sim 2$.

As is done with the luminosity functions of distant galaxies, it may thus be possible to use the form of the H II LF to understand features in the most recent star formation history ( $\lesssim 10 \mathrm{Myr}$ ) of their host galaxies, since individual bursts can leave their imprint on the H II LF. Our models, for example, show that an aged burst population is distinguishable from continuous creation of low- $N_{*}$ clusters (cf. Figures $2 k c, f$ and $4 p$ ). Feinstein (1997) also demonstrates effects of different creation histories. At present, it is difficult to make useful quantitative constraints, owing to present constraints on stellar and cluster parameters, but it is at least possible gain qualitative insight on the recent star formation history.

We thank Rob Kennicutt, Johan Knapen, and the referee, Carlos Feinstein, for comments on the manuscript. We also thank Johan Knapen for access to data for M100 in advance of publication, and John Beckman for an interesting discussion.

\section{REFERENCES}

Banfi M., Rampazzo R., Chincarini G., \& Henry R. B. C., 1993, AA, 280, 373

Beltrametti M., Tenorio-Tagle G., Yorke H.W. 1982, AA, 112, 1

Caldwell N., Kennicutt R., Phillips A.C., \& Schommer R.A., 1991, ApJ 370, 526 
Deharveng L., Caplan J., Lequeux J., Azzopardi M., Breysacher J., Tarenghi M., \& Westerlund B., 1988, AAS, 73, 407

Feinstein C., 1997, ApJS 112, 29

Kennicutt R. C., Edgar B. K., \& Hodge P. W., 1989, ApJ, 337, 761 (KEH)

Knapen J. H., 1997, MNRAS, submitted

Knapen J. H., Arnth-Jensen N., Cepa J., \& Beckman J. E., 1993, AJ, 106, 56

Leitherer C., 1990, ApJS, 73, 1

Leitherer C. \& Heckman T. M. 1995, ApJS, 96, 9

Massey P., Lang C. C., DeGioia-Eastwood K., \& Garmany C. D. 1995, ApJ, 438, 188

McKee C. F. \& Williams J. P. 1997, ApJ, 476, 144 (MW97)

Oey M. S. \& Clarke C. J. 1997, MNRAS, 289, 570

Panagia N. 1973, AJ, 78, 929

Press W. H., Flannery B. P., Teukolsky S. A., \& Vetterling W. T., 1986, Numerical Recipes, Cambridge: Cambridge Univ. Press, 195

Rand R. J., 1992, AJ, 103, 815

Rand R. J., 1993, ApJ, 410, 68

Rozas M., Beckman J. E., \& Knapen J. H. 1996a, AA, 307,735

Rozas M., Knapen J. H., \& Beckman J. E. 1996b, AA, 312, 275

Salpeter E. E. 1955, ApJ, 121, 161

Scalo J., 1986, Fund. Cosmic Phys., 11, 1

Schaerer D., de Koter A. 1997, AA, 322, 598

Schaerer D., Meynet G., Maeder A., \& Schaller G., 1993, AAS, 98, 523

Scowen, P. A., Dufour, R. J., \& Hester, J. J. 1992, AJ, 104, 92

Thronson H. A., Rubin H., \& Ksir A., 1991, MNRAS, 252,550

Vacca W. D., Garmany C. D., \& Shull J. M. 1996, ApJ, 460, 914 von Hippel T. \& Bothun G., 1990, AJ, 100, 403

Walterbos R. A. M. \& Braun R. 1992, ApJS, 92, 625 